\title{
Some Topological and Geometric Properties of Some New Spaces of $\lambda$-Convergent and Bounded Series
}

\author{
Meltem Kaya and Hasan Furkan \\ Department of Mathematics, Kahramanmaraş Sütçü İmam University, 46100 Kahramanmaraş, Turkey \\ Correspondence should be addressed to Hasan Furkan; hasanfurkan@hotmail.com
}

Received 16 March 2015; Accepted 14 April 2015

Academic Editor: Alberto Fiorenza

Copyright ( $) 2015$ M. Kaya and H. Furkan. This is an open access article distributed under the Creative Commons Attribution License, which permits unrestricted use, distribution, and reproduction in any medium, provided the original work is properly cited.

\begin{abstract}
The main purpose of this study is to introduce the spaces $c s^{\lambda}, c s_{0}^{\lambda}$, and $b s^{\lambda}$ which are $B K$-spaces of nonabsolute type. We prove that these spaces are linearly isomorphic to the spaces $c s, c s_{0}$, and $b s$, respectively, and derive some inclusion relations. Additionally, Schauder bases of the spaces $c s^{\lambda}$ and $c s_{0}^{\lambda}$ have been constructed and the $\alpha-, \beta$-, and $\gamma$-duals of these spaces have been computed. Besides, we characterize some matrix classes from the spaces $c s^{\lambda}, c s_{0}^{\lambda}$, and $b s^{\lambda}$ to the spaces $\ell_{p}, c$, and $c_{0}$, where $1 \leq p \leq \infty$. Finally, we examine some geometric properties of these spaces as Gurarìs modulus of convexity, property $m_{\infty}$, property $(M)$, property WORTH, nonstrict Opial property, and weak fixed point property.
\end{abstract}

\section{Introduction}

By a sequence space, we understand a linear subspace of the space $w=\mathbf{C}^{\mathbf{N}}$, where $\mathbf{N}=\{0,1,2, \ldots\}$ and $\mathbf{C}$ denotes the complex field. A sequence space $E$ with a linear topology is called a $K$-space provided each of the maps $p_{i}: E \rightarrow \mathbf{C}$ defined $p_{i}(x)=x_{i}$ which is continuous for all $i \in \mathbf{N}$. A $K$ space is called an $F K$-space provided $E$ is a complete linear metric space. An FK-space whose topology is normable is called a $B K$-space (see [1, pages 272-273]) which contains $\phi$, the set of all finitely nonzero sequences. We write $\ell_{\infty}, c$, and $c_{0}$ for the spaces of all bounded, convergent, and null sequences, respectively. Also by $\ell_{p}$, we denote the space of all $p$ absolutely summable sequences, where $1 \leq p<\infty$. Moreover, we write $b s, c s$, and $c s_{0}$ for the spaces of all bounded, convergent, and null series, respectively.

Let $\mu$ and $\nu$ be two sequence spaces, and let $A=\left(a_{n k}\right)$ be an infinite matrix of complex numbers $a_{n k}$, where $n, k \in \mathbf{N}$. Then we say that $A$ defines a matrix transformation from $\mu$ into $\nu$, and we denote it by writing $A: \mu \rightarrow \nu$ if for every sequence $x=\left(x_{k}\right) \in \mu$, the sequence $A x=\left\{(A x)_{n}\right\}$, the $A$ transform of $x$, is in $v$, where

$$
(A x)_{n}:=\sum_{k} a_{n k} x_{k}, \quad\left(n \in \mathbf{N}, x \in D_{00}(A)\right)
$$

and by $D_{00}(A)$ denotes the subspace of $w$ consisting of $x \in w$ for which the sum exists as a finite sum. For simplicity in notation, here and in what follows, the summation without limits runs from 0 to $\infty$ and we will use the convention that any term with a negative subscript is equal to naught; for example, $\lambda_{-1}=0$ and $x_{-1}=0$.

By $(\mu: \nu)$, we denote the class of all matrices $A$ such that $A: \mu \rightarrow \nu$. Thus $A \in(\mu: \nu)$ if and only if the series on the right side of (1) converges for each $n \in \mathbf{N}$ and each $x \in \mu$ and we have $A x=\left\{(A x)_{n}\right\}_{n \in \mathbf{N}} \in \nu$ for all $x \in \mu$. For an arbitrary sequence space $\mu$, the matrix domain $\mu_{A}$ of an infinite matrix $A$ in $\mu$ is defined by

$$
\mu_{A}:=\{x \in w: A x \in \mu\},
$$

which is a sequence space. If $A$ is triangle, then one can easily observe that the normed sequence spaces $\mu_{A}$ and $\mu$ are norm isomorphic; that is, $\mu_{A} \cong \mu$. If $\mu$ is a sequence space, then the continuous dual $\mu_{A}^{*}$ of the space $\mu_{A}$ is defined by

$$
\mu_{A}^{*}:=\left\{f: f=g \circ A, g \in \mu^{*}\right\} .
$$

We denote the collection of all finite subsets of $\mathbf{N}$ by $\mathscr{F}$. Also, we will write $e^{(k)}$ for the sequence whose only nonzero term is 1 in the $k$ th place for each $k \in \mathbf{N}$. Throughout this paper, 
let $\lambda=\left(\lambda_{k}\right)$ be a strictly increasing sequence of positive real numbers tending to infinity; that is,

$$
\begin{aligned}
0 & <\lambda_{0}<\lambda_{1}<\lambda_{2}<\cdots, \\
\lim _{k \rightarrow \infty} \lambda_{k} & =\infty .
\end{aligned}
$$

We define the matrix $\Lambda=\left(\lambda_{n k}\right)$ of weighted mean relative to the sequence $\lambda$ by

$$
\lambda_{n k}= \begin{cases}\frac{\lambda_{k}-\lambda_{k-1}}{\lambda_{n}}, & 0 \leq k \leq n, \\ 0, & k>n,\end{cases}
$$

for all $k ; n \in \mathbf{N}$. With a direct calculation we derive the equality

$$
(\Lambda x)_{n}=\frac{1}{\lambda_{n}} \sum_{k=0}^{n}\left(\lambda_{k}-\lambda_{k-1}\right) x_{k} ; \quad(n \in \mathbf{N}) .
$$

It is easy to show that the matrix $\Lambda$ is regular and is reduced, in the special case $\lambda_{k}=k+1$ for all $k \in \mathbf{N}$ to the matrix $C_{1}$ of Cesàro means of order one. Introducing the concept of $\Lambda$ strong convergence, several results on $\Lambda$-strong convergence of numerical sequences and Fourier series were given by Móricz [2]. Since we have in the special case

$$
\begin{aligned}
& Q_{n}=\sum_{k=0}^{n} q_{k}=\lambda_{n}, \\
& r_{n k}=\frac{q_{k}}{Q_{n}}=\frac{\lambda_{k}-\lambda_{k-1}}{\lambda_{n}}=\lambda_{n k},
\end{aligned}
$$

$q_{k}=\lambda_{k}-\lambda_{k-1}$ for all $k \in \mathbf{N}$, the matrix $\Lambda$ is also reduced to the Riesz means $R^{q}=\left(r_{n k}\right)$ with respect to the sequence $q=\left(q_{k}\right)$.

We summarize the knowledge in the existing literature concerning domain of the matrix $\lambda$ over some sequence spaces. Mursaleen and Noman [3-6] introduced the spaces $\ell_{\infty}^{\lambda}, c^{\lambda}, c_{0}^{\lambda}$, and $\ell_{p}^{\lambda}$ of lambda-bounded, lambda-convergent, lambda-null, and lambda-absolutely $p$-summable sequences and gave the inclusion relations between these spaces and the classical sequence spaces $\ell_{\infty}, c$, and $c_{0}$. Later, Mursaleen and Noman [7] investigated the difference spaces $c_{0}^{\lambda}(\Delta)$ and $c^{\lambda}(\Delta)$ obtained from the spaces $c_{0}^{\lambda}$ and $c^{\lambda}$. Recently, paranormed $\lambda$-sequence spaces of nonabsolute type have been studied by Karakaya et al. [8]. More recently, Sönmez and Başar [9] introduce the difference sequence spaces $c_{0}^{\lambda}(B)$ and $c^{\lambda}(B)$, which are the generalization of the spaces $c_{0}^{\lambda}(\Delta)$ and $c^{\lambda}(\Delta)$. Quite recently, some new sequence spaces of nonabsolute type and matrix transformations have been studied by Ganie and Sheikh [10]. The same authors have studied the spaces of $\lambda$-convergent sequences and almost convergence [11]. Also, the fine spectrum of the operator defined by lambda matrix over the spaces of null and convergent sequences has been studied by Yeşilkayagil and Başar [12].

In this work, our purpose is to construct the sequence spaces $c s_{0}^{\lambda}, c s^{\lambda}$, and $b s^{\lambda}$ by the domain of the matrix $\Lambda$ in the spaces $c s_{0}, c s$, and $b s$, respectively, of the series whose sequence of partial sums are in the spaces $c_{0}, c$, and $\ell_{\infty}$ [3].
We define the sequence $y=\left(y_{k}\right)$ by the $\Lambda$-transform $\Lambda x$ of a sequence $x=\left(x_{k}\right)$; that is, $y=\Lambda x$, and so we have

$$
y_{k}:=\frac{1}{\lambda_{k}} \sum_{j=0}^{k}\left(\lambda_{j}-\lambda_{j-1}\right) x_{j} ; \quad(k \in \mathbf{N}) .
$$

Also, we say that a sequence $x=\left(x_{k}\right) \in w$ is $\lambda$-convergent if $\Lambda x \in c$. In particular, we say that $x$ is $\lambda$-null or $\lambda$-bounded if $\Lambda x \in c_{0}$ or $\ell_{\infty}$, respectively.

\section{The Sequence Spaces $c s^{\lambda}, c s_{0}^{\lambda}$, and $b s^{\lambda}$}

In the present section, we introduce the sequence spaces $c s^{\lambda}$, $c s_{0}^{\lambda}$, and $b s^{\lambda}$ as the sets of all sequences whose $\Lambda$-transforms are in the spaces $c s, c s_{0}$, and $b s$, respectively; that is,

$$
\begin{aligned}
c s^{\lambda} & =\left\{x=\left(x_{k}\right)\right. \\
& \left.\in w: \lim _{m \rightarrow \infty} \sum_{n=0}^{m} \frac{1}{\lambda_{n}} \sum_{k=0}^{n}\left(\lambda_{k}-\lambda_{k-1}\right) x_{k} \text { exists }\right\}, \\
c s_{0}^{\lambda} & =\left\{x=\left(x_{k}\right)\right. \\
& \left.\in w: \lim _{m \rightarrow \infty} \sum_{n=0}^{m} \frac{1}{\lambda_{n}} \sum_{k=0}^{n}\left(\lambda_{k}-\lambda_{k-1}\right) x_{k}=0\right\}, \\
b s^{\lambda} & =\left\{x=\left(x_{k}\right) \in w: \sup _{m}\left|\sum_{n=0}^{m} \frac{1}{\lambda_{n}} \sum_{k=0}^{n}\left(\lambda_{k}-\lambda_{k-1}\right) x_{k}\right|\right. \\
& <\infty\} .
\end{aligned}
$$

With the notation of (2), we can redefine the spaces $c s^{\lambda}$, $c s_{0}^{\lambda}$, and $b s^{\lambda}$ as the matrix domains of the triangle $\Lambda$ in the spaces $c s, c s_{0}$, and $b s$ by

$$
\begin{aligned}
& c s^{\lambda}=(c s)_{\Lambda}, \\
& c s_{0}^{\lambda}=\left(c s_{0}\right)_{\Lambda}, \\
& b s^{\lambda}=(b s)_{\Lambda} .
\end{aligned}
$$

Then, it is immediate by (12) that the sets $c s^{\lambda}, c s_{0}^{\lambda}$, and $b s^{\lambda}$ are linear spaces with coordinatewise addition and scalar multiplication; that is, $c s^{\lambda}, c s_{0}^{\lambda}$, and $b s^{\lambda}$ are the sequence spaces consisting of all sequences which are $\lambda$-convergent, $\lambda$ null, and $\lambda$-bounded series of type $\lambda$, respectively.

Now, we may begin with the following theorem which is essential in the text.

Theorem 1. The sequence spaces $c s^{\lambda}, c s_{0}^{\lambda}$, and $b s^{\lambda}$ are $B K$ spaces with the same norm $\|x\|_{\mathcal{c s}^{\lambda}}=\|x\|_{c s_{0}^{\lambda}}=\|x\|_{b s^{\lambda}}$; that is,

$$
\|x\|_{b s^{\lambda}}=\|\Lambda x\|_{b s}=\sup _{m}\left|\sum_{n=0}^{m}(\Lambda x)_{n}\right|<\infty .
$$


Proof. Since (12) holds and $c s, c s_{0}$, and $b s$ are $B K$-spaces with the respect to their natural norms and the matrix $\Lambda$ is a triangle, Theorem 4.3.12 of Wilansky [13, page 63] gives the fact that $c s^{\lambda}, c s_{0}^{\lambda}$, and $b s^{\lambda}$ are $B K$-spaces with the given norms. This completes the proof.

Remark 2. One can easily check that the absolute property does not hold on the spaces $c s^{\lambda}, c s_{0}^{\lambda}$, and $b s^{\lambda}$; that is, $\|x\|_{c s^{\lambda}} \neq$ $\||x|\|_{c s^{\lambda}},\|x\|_{c s_{0}^{\lambda}} \neq\||x|\|_{c s_{0}^{\lambda}}$, and $\|x\|_{b s^{\lambda}} \neq\||x|\|_{b s^{\lambda}}$ for at least one sequence in the spaces $c s^{\lambda}, c s_{0}^{\lambda}$, and $b s^{\lambda}$, and this shows that $c s^{\lambda}, c s_{0}^{\lambda}$, and $b s^{\lambda}$ are the sequence spaces of nonabsolute type, where $|x|=\left(\left|x_{k}\right|\right)$.

Now, we give the final theorem of this section.

Theorem 3. The sequence spaces $c s^{\lambda}, c s_{0}^{\lambda}$, and $b s^{\lambda}$ of nonabsolute type are isometrically isomorphic to the spaces $c s, c s_{0}$, and $b s$, respectively; that is, $c s^{\lambda} \cong c s, c s_{0}^{\lambda} \cong c s_{0}$, and $b s^{\lambda} \cong b s$.

Proof. To prove this, we should show the existence of an isometric isomorphism between the spaces $c s_{0}^{\lambda}$ and $c s_{0}$. Consider the transformation $T$ defined, with the notation of (8), from $c s_{0}^{\lambda}$ to $c s_{0}$ by $x \mapsto y=T x$. Then, $T x=y=\Lambda x \in c s_{0}$ for every $x \in c s_{0}^{\lambda}$ and the linearity of $T$ is clear. Also, it is trivial that $x=\theta$ whenever $T x=\theta$ and hence $T$ is injective. Furthermore, let $y=\left(y_{k}\right) \in c s_{0}$ be given and define the sequence $x=\left(x_{k}\right)$ by

$$
x_{k}:=\sum_{j=k-1}^{k}(-1)^{k-j} \frac{\lambda_{j}}{\lambda_{k}-\lambda_{k-1}} y_{j} ; \quad(k \in \mathbf{N}) .
$$

Then, by using (8) and (14), we have for every $n \in \mathbf{N}$ that

$$
\begin{aligned}
(\Lambda x)_{n} & =\frac{1}{\lambda_{n}} \sum_{k=0}^{n}\left(\lambda_{k}-\lambda_{k-1}\right) x_{k} \\
& =\frac{1}{\lambda_{n}} \sum_{k=0}^{n} \sum_{j=k-1}^{k}(-1)^{k-j} \lambda_{j} y_{j} \\
& =\frac{1}{\lambda_{n}} \sum_{k=0}^{n}\left(\lambda_{k} y_{k}-\lambda_{k-1} y_{k-1}\right)=y_{n} .
\end{aligned}
$$

This shows that $\Lambda x=y$ and since $y \in c s_{0}$, we obtain that $\Lambda x \in c s_{0}$. Thus, we deduce that $x \in c s_{0}^{\lambda}$ and $T x=y$. Hence $T$ is surjective. Moreover, one can easily see for every $x \in c s_{0}^{\lambda}$ that

$$
\|T x\|_{\mathcal{H S}_{0}}=\|x\|_{\mathcal{c s}_{0}^{\lambda}},
$$

which means that $T$ is norm preserving. Therefore $T$ is isometry. Consequently $T$ is an isometric isomorphism which shows that the spaces $c s_{0}^{\lambda}$ and $c s_{0}$ are isometrically isomorphic.

It is clear that if the spaces $c s_{0}^{\lambda}$ and $c s_{0}$ are replaced by the respective one of the spaces $c s^{\lambda}$ and $c s$ or $b s^{\lambda}$ and $b s$, then we obtain the fact that $c s^{\lambda} \cong c s$ and $b s^{\lambda} \cong b s$. This completes the proof.

\section{The Inclusion Relations}

In the present section, we establish some inclusion relations concerning the spaces $c s^{\lambda}, c s_{0}^{\lambda}$, and $b s^{\lambda}$. We may begin with the following lemma.

Lemma 4 (see [3]). For any sequence $x=\left(x_{k}\right) \in w$, the equality

$$
(S x)_{n}=x_{n}-(\Lambda x)_{n} ; \quad(n \in \mathbf{N})
$$

holds, where $S x=\left\{(S x)_{n}\right\}$ is the sequence defined by

$$
\begin{aligned}
& (S x)_{0}=0, \\
& (S x)_{n}=\frac{1}{\lambda_{n}} \sum_{k=1}^{n} \lambda_{k-1}\left(x_{k}-x_{k-1}\right) ; \quad(n \geq 1) .
\end{aligned}
$$

Theorem 5. The inclusions $c s_{0}^{\lambda} \subset c s^{\lambda} \subset b s^{\lambda}$ strictly hold.

Proof. It is obvious that the inclusions $c s_{0}^{\lambda} c c s^{\lambda} c b s^{\lambda}$ hold. Let us consider the sequence $x=\left(x_{k}\right)$ defined by

$$
x_{k}=\frac{\lambda_{k}\left(1 /(k+2)^{2}\right)-\lambda_{k-1}\left(1 /(k+1)^{2}\right)}{\lambda_{k}-\lambda_{k-1}} ;
$$

$(k \in \mathbf{N})$

In the present case, we obtain for every $n \in \mathbf{N}$ that

$$
\begin{aligned}
(\Lambda x)_{n} & =\frac{1}{\lambda_{n}} \sum_{k=0}^{n}\left(\lambda_{k} \frac{1}{(k+2)^{2}}-\lambda_{k-1} \frac{1}{(k+1)^{2}}\right) \\
& =\frac{1}{(n+2)^{2}},
\end{aligned}
$$

which shows that $\Lambda x \in c s \backslash c s_{0}$. Thus, the sequence $x$ is in $c s^{\lambda}$ but not in $c s_{0}^{\lambda}$. Hence $c s_{0}^{\lambda} c c s^{\lambda}$ is a strict inclusion.

To show the strictness of the inclusion $c s^{\lambda} c b s^{\lambda}$, we define the sequence $y=\left(y_{k}\right)$ by

$$
y_{k}=(-1)^{k}\left(\frac{\lambda_{k}+\lambda_{k-1}}{\lambda_{k}-\lambda_{k-1}}\right) ; \quad(k \in \mathbf{N}) .
$$

Then, we have for every $m \in \mathbf{N}$ that

$$
\sum_{n=0}^{m}(\Lambda y)_{n}=\sum_{n=0}^{m} \frac{1}{\lambda_{n}} \sum_{k=0}^{n}(-1)^{k}\left(\lambda_{k}+\lambda_{k-1}\right)=\sum_{n=0}^{m}(-1)^{n} .
$$

This shows $\Lambda y \in b s \backslash c s$. Thus, the sequence $y$ is in $b s^{\lambda}$ but not in $c s^{\lambda}$ and hence $c s^{\lambda} c b s^{\lambda}$ is a strict inclusion. This concludes the proof.

Lemma 6 (see [3, Theorem 4.1.]). The inclusions $c_{0}^{\lambda} \subset c^{\lambda} \subset \ell_{\infty}^{\lambda}$ strictly hold.

Theorem 7. The inclusions $c s^{\lambda} \subset c_{0}^{\lambda}$ and $b s^{\lambda} \subset \ell_{\infty}^{\lambda}$ strictly hold. 
Proof. It is clear that the inclusion $c s^{\lambda} \subset c_{0}^{\lambda}$ holds, since $x \in$ $c s^{\lambda}$ implies $\Lambda x \in c s$ and hence $\Lambda x \in c_{0}$ which means that $x \in c_{0}^{\lambda}$. Consider the sequence $x=\left(x_{k}\right)$ defined by

$$
x_{k}=\frac{1}{k+1} ; \quad(k \in \mathbf{N}) \text {. }
$$

Then, $x \in c_{0}$ and hence $x \in c_{0}^{\lambda}$, since the inclusion $c_{0} \subset c_{0}^{\lambda}$ holds. On the other hand, we have for every $n \in \mathbf{N}$ that

$$
\begin{aligned}
(\Lambda x)_{n} & =\frac{1}{\lambda_{n}} \sum_{k=0}^{n} \frac{\lambda_{k}-\lambda_{k-1}}{k+1} \\
& \geq \frac{1}{\lambda_{n}(n+1)} \sum_{k=0}^{n}\left(\lambda_{k}-\lambda_{k-1}\right)=\frac{1}{n+1},
\end{aligned}
$$

which shows that $\Lambda x \notin c s$ and hence $x \notin c s^{\lambda}$. Thus, the sequence $x$ is in $c_{0}^{\lambda}$ but not in $c s^{\lambda}$. Therefore, the inclusion $c s^{\lambda} c c_{0}^{\lambda}$ is strict.

Similarly, it is also trivial that the inclusion $b s^{\lambda} \subset \ell_{\infty}^{\lambda}$ holds. To show that this inclusion is strict, we define the sequence $y=\left(y_{k}\right)$ by $y=e=(1,1,1, \ldots)$. In the present case, we have for every $n \in \mathbf{N}$ that

$$
(\Lambda y)_{n}=\frac{1}{\lambda_{n}} \sum_{k=0}^{n}\left(\lambda_{k}-\lambda_{k-1}\right)=1,
$$

which shows that $\Lambda y \in \ell_{\infty} \backslash b s$. Thus, the sequence $y$ is in $\ell_{\infty}^{\lambda}$ but not in $b s^{\lambda}$ and hence $b s^{\lambda} \subset \ell_{\infty}^{\lambda}$ is a strict inclusion. This completes the proof.

Theorem 8. The inclusion $c s^{\lambda} c c s$ holds if and only if $S x \in c s$ for every sequence $x \in c s^{\lambda}$.

Proof. Suppose that the inclusion $c s^{\lambda} c c s$ holds, and take any $x=\left(x_{k}\right) \in c s^{\lambda}$. Then $\Lambda x \in c s$ and $x \in c s$ by the hypothesis. Thus, we deduce from (17) that

$$
\sum_{n=0}^{m}\left(x_{n}-(\Lambda x)_{n}\right)=\sum_{n=0}^{m} x_{n}-\sum_{n=0}^{m}(\Lambda x)_{n}=\sum_{n=0}^{m}(S x)_{n} .
$$

Hence, we obtain from (26) by letting $m \rightarrow \infty$ that

$$
\lim _{m} \sum_{n=0}^{m}(S x)_{n}=\sum_{n} x_{n}-\sum_{n}(\Lambda x)_{n} .
$$

As $\left(x_{n}\right) \in c s$ and $\left((\Lambda x)_{n}\right) \in c s$, the right-hand side of equality (27) is convergent as $m \rightarrow \infty$. Thereby, the series $\sum_{n=0}^{m}(S x)_{n}$ converges and so $S x \in c s$.

Conversely, let $x \in c s^{\lambda}$ be given. Then, we have by the hypothesis that $S x \in c s$. Again, it follows by (26) that

$$
\lim _{m} \sum_{n=0}^{m} x_{n}=\sum_{n}(S x)_{n}+\sum_{n}(\Lambda x)_{n}
$$

which shows that $x \in c s$ while $\Lambda x \in c s$ and $S x \in c s$. Hence, the inclusion $c s^{\lambda} \subset c s$ holds and this concludes the proof.
Theorem 9. The inclusion $c s_{0}^{\lambda} \subset c s_{0}$ holds if and only if $S x \in$ $c s_{0}$ for every sequence $x \in c s_{0}^{\lambda}$.

Proof. One can see by analogy to Theorem 8 that the inclusion $c s_{0}^{\lambda} \subset c s_{0}$ also holds if and only if $S x \in c s_{0}$ for every sequence $x \in c s_{0}^{\lambda}$. This completes the proof.

Theorem 10. The inclusion $b s^{\lambda} \subset$ bs holds if and only if $S x \in$ bs for every sequence $x \in b s^{\lambda}$.

Proof. Suppose that the inclusion $b s^{\lambda} \subset$ bs holds, and take any $x=\left(x_{k}\right) \in b s^{\lambda}$. Then, $x \in b s$ by the hypothesis. Thus, we obtain from equality (17)

$$
\|S x\|_{b s} \leq\|x\|_{b s}+\|\Lambda x\|_{b s}=\|x\|_{b s}+\|x\|_{b s^{\lambda}}<\infty,
$$

which yields that $S x \in b s$.

Conversely, assume that $S x \in b s$ for every $x \in b s^{\lambda}$. Again, we obtain from equality (17)

$$
\|x\|_{b s} \leq\|S x\|_{b s}+\|\Lambda x\|_{b s}=\|S x\|_{b s}+\|x\|_{b s^{\lambda}}<\infty .
$$

This shows that $x \in b s$. Hence, the inclusion $b s^{\lambda} c b s$ holds. This completes the proof.

\section{The Basis for the Spaces $c s^{\lambda}, c s_{0}^{\lambda}$, and $b s^{\lambda}$}

In the present section, we give a sequence of the points of the spaces $c s^{\lambda}$ and $c s_{0}^{\lambda}$ which forms a basis for these spaces. If a normed sequence space $X$ contains a sequence $\left(b_{n}\right)$ with the property that for every $x \in X$ there is a unique sequence of scalars $\left(\alpha_{n}\right)$ such that

$$
\lim _{n \rightarrow \infty}\left\|x-\left(\alpha_{0} b_{0}+\alpha_{1} b_{1}+\cdots+\alpha_{n} b_{n}\right)\right\|=0
$$

then $\left(b_{n}\right)$ is called a Schauder basis (or briefly basis) for $X$. The series $\sum_{k} \alpha_{k} b_{k}$ which has the sum $x$ is then called the expansion of $x$ with respect to $\left(b_{n}\right)$ and is written as $x=$ $\sum_{k} \alpha_{k} b_{k}$.

Now, since the transformation $T$ defined from $c s_{0}^{\lambda}$ to $c s_{0}$ in the proof of Theorem 3 is an isomorphism, we have the following theorem.

Theorem 11. Define the sequence $e_{\lambda}^{(n)}=\left\{\left(e_{\lambda}^{(n)}\right)_{k}\right\}$ for every fixed $k \in \mathbf{N}$ by

$$
\left\{\left(e_{\lambda}^{(n)}\right)_{k}\right\}= \begin{cases}(-1)^{k-n} \frac{\lambda_{n}}{\lambda_{k}-\lambda_{k-1}}, & n \leq k \leq n+1, \\ 0, & \text { otherwise, }\end{cases}
$$

for all $k \in \mathbf{N}$.

Then, one has the following:

(a) The sequence $\left(e_{\lambda}^{(0)}, e_{\lambda}^{(1)}, \ldots\right)$ is a Schauder basis for the spacescs ${ }^{\lambda}$ and $c s_{0}^{\lambda}$ and every $x \in \operatorname{cs}^{\lambda} \operatorname{orcs}_{0}^{\lambda}$ has a unique representation of the form

$$
x=\sum_{n=0}^{\infty}(\Lambda x)_{n} e_{\lambda}^{(n)} .
$$

(b) $b s^{\lambda}$ has no Schauder basis. 
Proof. (a) It is clear that $e_{\lambda}^{(n)}$ is a basis for $c s^{\lambda}$ since $e^{(n)}$ is a basis for $c s$ and $\Lambda\left(e_{\lambda}^{(n)}\right)=e^{(n)}\left[14\right.$, Corollary 2.3]. Let $x \in c s^{\lambda}$ be given. Then, $y=\Lambda x \in c s$ and

$$
y^{[m]}=\sum_{n=0}^{m} y_{n} e^{(n)} \longrightarrow y \quad(m \longrightarrow \infty)
$$

for a unique sequence $\left(y_{n}\right)_{n=0}^{\infty}$ of scalars. Therefore, we obtain that

$$
\widehat{\Lambda}\left(y^{[m]}\right)=\sum_{n=0}^{m} y_{n} \widehat{\Lambda}\left(e^{(n)}\right)=\sum_{n=0}^{m} y_{n} e_{\lambda}^{(n)},
$$

where $\widehat{\Lambda}$ is the inverse of the matrix $\Lambda$. Since $y_{n}=(\Lambda x)_{n}$, we get that

$$
x^{[m]}=\sum_{n=0}^{m}(\Lambda x)_{n} e_{\lambda}^{(n)} .
$$

Consequently,

$$
\begin{aligned}
\left\|x^{[m]}-x\right\|_{c s^{\lambda}} & =\left\|\Lambda\left(x^{[m]}-x\right)\right\|_{\mathcal{C S}}=\left\|\Lambda\left(x^{[m]}\right)-\Lambda x\right\|_{\mathcal{C S}} \\
& =\left\|y^{[m]}-y\right\|_{\mathcal{C S}} \longrightarrow 0 \quad(m \longrightarrow \infty) .
\end{aligned}
$$

Thus, we deduce that $\lim _{m \rightarrow \infty}\left\|x^{[m]}-x\right\|_{c s^{\lambda}}=0$, which shows that $x \in c s^{\lambda}$ is represented as in (33).

Finally, let us show the uniqueness of the representation (33) of $x \in c s^{\lambda}$. Suppose on the contrary that there exists another representation $x=\sum_{n} \alpha_{n} e_{\lambda}^{(n)}$. Since the linear transformation $T$ defined from $c s^{\lambda}$ to $c s$, in the proof of Theorem 3 , is continuous, we have

$$
(\Lambda x)_{k}=\sum_{n} \alpha_{n}\left(\Lambda e_{\lambda}^{(n)}\right)_{k}=\sum_{n} \alpha_{n} \delta_{k n}=\alpha_{k} ; \quad(k \in \mathbf{N}) .
$$

Therefore, the representation (33) of $x \in c s^{\lambda}$ is unique. It can be proved similarly for $c s_{0}^{\lambda}$. This completes the proof.

(b) As a direct consequence of Remark 2.2. of Malkowsky and Rakocevic [14], since $b s$ has no Schauder basis $b s^{\lambda}$ also has no Schauder basis.

As a result, it easily follows from Theorem 1 that $c s^{\lambda}$ and $c s_{0}^{\lambda}$ are the Banach spaces with their natural norms. Then, by Theorem 11 we obtain the following corollary.

Corollary 12. The sequence spaces $c s^{\lambda}$ and $c s_{0}^{\lambda}$ of nonabsolute type are separable.

\section{The $\alpha$-, $\beta$-, and $\gamma$-Duals of the Spaces $c s^{\lambda}, c s_{0}^{\lambda}$, and $b s^{\lambda}$}

In this section, we state and prove the theorems determining the $\alpha$-, $\beta$-, and $\gamma$-duals of the sequence spaces $c s^{\lambda}, c s_{0}^{\lambda}$, and $b s^{\lambda}$ of nonabsolute type. For arbitrary sequence spaces $X$ and $Y$, the set $M(X, Y)$ defined by

$$
\begin{aligned}
& M(X, Y) \\
& \quad=\left\{a=\left(a_{k}\right) \in w: a x=\left(a_{k} x_{k}\right) \in Y \forall x=\left(x_{k}\right) \in X\right\}
\end{aligned}
$$

is called the multiplier space of $X$ and $Y$. One can easily observe for a sequence space $Z$ with $Y \subset Z$ and $Z \subset X$ that the inclusions $M(X, Y) \subset M(X, Z)$ and $M(X, Y) \subset M(Z, Y)$ hold, respectively. With the notation of (39), the $\alpha$-, $\beta$-, and $\gamma$ duals of a sequence space $X$, which are, respectively, denoted by $X^{\alpha}, X^{\beta}$, and $X^{\gamma}$, are defined by

$$
\begin{aligned}
& X^{\alpha}=M\left(X, \ell_{1}\right), \\
& X^{\beta}=M(X, c s), \\
& X^{\gamma}=M(X, b s) .
\end{aligned}
$$

It is clear that $X^{\alpha} \subset X^{\beta} \subset X^{\gamma}$. Also, it can be obviously seen that the inclusions $X^{\alpha} \subset Y^{\alpha}, X^{\beta} \subset Y^{\beta}$, and $X^{\gamma} \subset Y^{\gamma}$ hold whenever $Y \subset X$.

The following known results [15] are fundamental for this section.

Lemma 13. Consider $A=\left(a_{n k}\right) \in\left(c s: \ell_{1}\right)$ if and only if

$$
\sup _{N, K \in \mathscr{F}}\left|\sum_{n \in N} \sum_{k \in K}\left(a_{n k}-a_{n, k-1}\right)\right|<\infty .
$$

Lemma 14. Consider $A=\left(a_{n k}\right) \in\left(c s_{0}: \ell_{1}\right)$ if and only if

$$
\sup _{N, K \in \mathscr{F}}\left|\sum_{n \in N} \sum_{k \in K}\left(a_{n k}-a_{n, k+1}\right)\right|<\infty .
$$

Lemma 15. Consider $A=\left(a_{n k}\right) \in\left(b s: \ell_{1}\right)$ if and only if (42) holds and

$$
\lim _{k} a_{n k}=0, \quad \forall n \in \mathbf{N} .
$$

Lemma 16. Consider $A=\left(a_{n k}\right) \in(c s: c)$ if and only if

$$
\begin{aligned}
& \sup _{n} \sum_{k}\left|a_{n k}-a_{n, k+1}\right|<\infty, \\
& \lim _{n} a_{n k} \text { exists } \forall k \in \mathbf{N} .
\end{aligned}
$$

Lemma 17. Consider $A=\left(a_{n k}\right) \in\left(c s_{0}: c\right)$ if and only if (44) holds:

$$
\lim _{n}\left(a_{n k}-a_{n, k+1}\right) \quad \text { exists } \forall k \in \mathbf{N} \text {. }
$$

Lemma 18. Consider $A=\left(a_{n k}\right) \in(b s: c)$ if and only if (43) and (45) hold and

$$
\sum_{k}\left|a_{n k}-a_{n, k-1}\right| \quad \text { converges. }
$$

Lemma 19. Consider $A=\left(a_{n k}\right) \in\left(c s: \ell_{\infty}\right)$ if and only if

$$
\sup _{n} \sum_{k}\left|a_{n k}-a_{n, k-1}\right|<\infty
$$

Lemma 20. Consider $A=\left(a_{n k}\right) \in\left(c s_{0}: \ell_{\infty}\right)$ if and only if (44) holds.

Lemma 21. Consider $A=\left(a_{n k}\right) \in\left(b s: \ell_{\infty}\right)$ if and only if (43) and (44) hold. 
Now, we prove the following result.

Theorem 22. Define the sets $m_{1}^{\lambda}$ and $m_{2}^{\lambda}$ as follows:

$$
\begin{aligned}
m_{1}^{\lambda} & =\left\{a=\left(a_{n}\right) \in w: \sup _{N, K \in \mathscr{F}}\left|\sum_{n \in N} \sum_{k \in K}\left(b_{n k}^{\lambda}-b_{n, k-1}^{\lambda}\right)\right|\right. \\
& <\infty\}, \\
m_{2}^{\lambda} & =\left\{a=\left(a_{n}\right) \in w: \sup _{N, K \in \mathscr{F}}\left|\sum_{n \in N} \sum_{k \in K}\left(b_{n k}^{\lambda}-b_{n, k+1}^{\lambda}\right)\right|\right. \\
& <\infty\},
\end{aligned}
$$

where the matrix $B^{\lambda}=\left(b_{n k}^{\lambda}\right)$ is defined via the sequence $a=$ $\left(a_{n}\right) \in w$ by

$$
\begin{aligned}
& b_{n k}^{\lambda} \\
& \quad= \begin{cases}(-1)^{n-k} \frac{\lambda_{k}}{\lambda_{n}-\lambda_{n}-1} a_{n} & \text { if } n-1 \leq k \leq n, \\
0 & \text { if } n-1>k \text { or } k>n,\end{cases}
\end{aligned}
$$

for all $n, k \in \mathbf{N}$. Then $\left\{c s^{\lambda}\right\}^{\alpha}=m_{1}^{\lambda}$ and $\left\{c s_{0}^{\lambda}\right\}^{\alpha}=\left\{b s^{\lambda}\right\}^{\alpha}=m_{2}^{\lambda}$.

Proof. Let $a=\left(a_{n}\right) \in w$. Then, by bearing in mind relations (8) and (14), it is immediate that the equality

$$
a_{n} x_{n}=\sum_{k=n-1}^{n}(-1)^{n-k} \frac{\lambda_{k}}{\lambda_{n}-\lambda_{n-1}} a_{n} y_{k}=\left(B^{\lambda} y\right)_{n}
$$

holds for all $n \in \mathbf{N}$. We therefore observe by (51) that $a x=$ $\left(a_{n} x_{n}\right) \in \ell_{1}$ whenever $x=\left(x_{k}\right) \in c s^{\lambda}$ if and only if $B^{\lambda} y \in \ell_{1}$ whenever $y=\left(y_{k}\right) \in c s$. This means that the sequence $a=$ $\left(a_{n}\right) \in\left\{c s^{\lambda}\right\}^{\alpha}$ if and only if $B^{\lambda} \in\left(c s: \ell_{1}\right)$. Hence, we obtain by Lemma 13 with $B^{\lambda}$ instead of $A$ that $a=\left(a_{n}\right) \in\left\{c s^{\lambda}\right\}^{\alpha}$ if and only if

$$
\sup _{N, K \in \mathscr{F}}\left|\sum_{n \in N} \sum_{k \in K}\left(b_{n k}^{\lambda}-b_{n, k-1}^{\lambda}\right)\right|<\infty
$$

which yields the result that $\left\{c s^{\lambda}\right\}^{\alpha}=m_{1}^{\lambda}$.

Similarly, we deduce from Lemma 15 with (51) that $a=$ $\left(a_{n}\right) \in\left\{b s^{\lambda}\right\}^{\alpha}$ if and only if $B^{\lambda} \in\left(b s: \ell_{1}\right)$. Then, it is clear that the columns of the matrix $B$ are in the space $c_{0}$, since

$$
\lim _{k} b_{n k}^{\lambda}=0
$$

for all $n \in \mathbf{N}$. Therefore, we derive from (42) that

$$
\sup _{N, K \in \mathscr{F}}\left|\sum_{n \in N} \sum_{k \in K}\left(b_{n k}^{\lambda}-b_{n, k+1}^{\lambda}\right)\right|<\infty .
$$

This shows that $\left\{c s_{0}^{\lambda}\right\}^{\alpha}=\left\{b s^{\lambda}\right\}^{\alpha}=m_{2}^{\lambda}$. This completes the proof.
Theorem 23. Define the sets $m_{3}^{\lambda}, m_{4}^{\lambda}$, and $m_{5}^{\lambda}$ as follows:

$$
\begin{aligned}
m_{3}^{\lambda} & =\left\{a=\left(a_{k}\right) \in w: \sum_{k=0}^{\infty}\left|\bar{\Delta}\left(\bar{\Delta}\left(\frac{a_{k}}{\lambda_{k}-\lambda_{k-1}}\right) \lambda_{k}\right)\right|\right. \\
& <\infty\}, \\
m_{4}^{\lambda} & =\left\{a=\left(a_{k}\right) \in w: \sup _{k}\left|\frac{\lambda_{k}}{\lambda_{k}-\lambda_{k-1}} a_{k}\right|<\infty\right\}, \\
m_{5}^{\lambda} & =\left\{a=\left(a_{k}\right) \in w: \lim _{k \rightarrow \infty} \frac{\lambda_{k}}{\lambda_{k}-\lambda_{k-1}} a_{k}=0\right\},
\end{aligned}
$$

where

$$
\bar{\Delta}\left(\frac{a_{k}}{\lambda_{k}-\lambda_{k-1}}\right)=\frac{a_{k}}{\lambda_{k}-\lambda_{k-1}}-\frac{a_{k+1}}{\lambda_{k+1}-\lambda_{k}}
$$

for all $k \in \mathbf{N}$. Then $\left\{c s^{\lambda}\right\}^{\beta}=\left\{c s_{0}^{\lambda}\right\}^{\beta}=m_{3}^{\lambda} \cap m_{4}^{\lambda}$ and $\left\{b s^{\lambda}\right\}^{\beta}=$ $m_{3}^{\lambda} \cap m_{5}^{\lambda}$.

Proof. Because the proof may also be obtained for the space $b s^{\lambda}$ in a similar way, we omit it. Take any $a=\left(a_{k}\right) \in w$ and consider the equation

$$
\begin{aligned}
\sum_{k=0}^{n} a_{k} x_{k} & =\sum_{k=0}^{n}\left[\sum_{j=k-1}^{k}(-1)^{k-j} \frac{\lambda_{j}}{\lambda_{k}-\lambda_{k-1}} y_{j}\right] a_{k} \\
& =\sum_{k=0}^{n-1} \bar{\Delta}\left(\frac{a_{k}}{\lambda_{k}-\lambda_{k-1}}\right) \lambda_{k} y_{k}+\frac{\lambda_{n}}{\lambda_{n}-\lambda_{n-1}} a_{n} y_{n} \\
& =\left(T^{\lambda} y\right)_{n},
\end{aligned}
$$

where the matrix $T^{\lambda}=\left(t_{n k}^{\lambda}\right)$ is defined by

$$
t_{n k}^{\lambda}= \begin{cases}\bar{\Delta}\left(\frac{a_{k}}{\lambda_{k}-\lambda_{k-1}}\right) \lambda_{k} & \text { if } 0 \leq k \leq n-1, \\ \frac{\lambda_{n}-a_{n}}{\lambda_{n}-\lambda_{n-1}} & \text { if } k=n, \\ 0 & \text { if } k>n,\end{cases}
$$

for all $n, k \in \mathbf{N}$. Thus, we deduce by (57) that $a x=\left(a_{k} x_{k}\right) \in c s$ where $x=\left(x_{k}\right) \in c s^{\lambda}$ if and only if $T^{\lambda} y \in c$ whenever $y=$ $\left(y_{k}\right) \in c s$. This means that $a=\left(a_{k}\right) \in\left\{c s^{\lambda}\right\}^{\beta}$ if and only if $T^{\lambda} \in(c s: c)$. Therefore, by using Lemma 16, we derive from (44) and (45) that

$$
\begin{aligned}
\sum_{k=0}^{\infty}\left|\bar{\Delta}\left(\bar{\Delta}\left(\frac{a_{k}}{\lambda_{k}-\lambda_{k-1}}\right) \lambda_{k}\right)\right| & <\infty, \\
\sup _{n}\left|\frac{\lambda_{n}}{\lambda_{n}-\lambda_{n-1}} a_{n}\right| & <\infty, \\
\lim _{n} t_{n k}^{\lambda} & =\bar{\Delta}\left(\frac{a_{k}}{\lambda_{k}-\lambda_{k-1}}\right) \lambda_{k},
\end{aligned}
$$

respectively. Thereby, we conclude that $\left\{c s^{\lambda}\right\}^{\beta}=\left\{c s_{0}^{\lambda}\right\}^{\beta}=$ $m_{3}^{\lambda} \cap m_{4}^{\lambda}$. 
Theorem 24. The $\gamma$-dual of the spaces $c s^{\lambda}, c s_{0}^{\lambda}$, and $b s^{\lambda}$ is the set $m_{3}^{\lambda} \cap m_{4}^{\lambda}$.

Proof. The proof of this result follows the same lines as those in the proof of Theorem 23 using Lemmas 19, 20, and 21 instead of Lemma 16.

\section{Certain Matrix Mappings on the Spaces $c s^{\lambda}, c s_{0}^{\lambda}$, and $b s^{\lambda}$}

In this present section, we characterize the matrix classes $\left(c s^{\lambda}: \ell_{p}\right),\left(c s_{0}^{\lambda}: \ell_{p}\right),\left(b s^{\lambda}: \ell_{p}\right),\left(c s^{\lambda}: c_{0}\right),\left(c s_{0}^{\lambda}: c_{0}\right),\left(b s^{\lambda}: c_{0}\right)$, $\left(c s^{\lambda}: c\right),\left(c s_{0}^{\lambda}: c\right)$, and $\left(b s^{\lambda}: c\right)$, where $1 \leq p \leq \infty$.

For an infinite matrix $A=\left(a_{n k}\right)$, we write for brevity that

$$
\begin{aligned}
\widetilde{a}_{n k} & =\bar{\Delta}\left(\frac{a_{n k}}{\lambda_{k}-\lambda_{k-1}}\right) \lambda_{k} \\
& =\left(\frac{a_{n k}}{\lambda_{k}-\lambda_{k-1}}-\frac{a_{n, k+1}}{\lambda_{k+1}-\lambda_{k}}\right) \lambda_{k} \quad(n, k \in \mathbf{N}) .
\end{aligned}
$$
results.

The following lemmas [15] will be needed in proving our

Lemma 25. Consider $A=\left(a_{n k}\right) \in\left(c s: c_{0}\right)$ if and only if (44) holds and

$$
\lim _{n} a_{n k}=0 \quad(\forall k \in \mathbf{N}) .
$$

Lemma 26. Consider $A=\left(a_{n k}\right) \in\left(c s_{0}: c_{0}\right)$ if and only if (44) holds and

$$
\lim _{n}\left(a_{n k}-a_{n, k+1}\right)=0 \quad(\forall k \in \mathbf{N}) .
$$

Lemma 27. Consider $A=\left(a_{n k}\right) \in\left(b s: c_{0}\right)$ if and only if (43) holds and

$$
\lim _{n} \sum_{k}\left|a_{n k}-a_{n, k+1}\right|=0
$$

Lemma 28. Consider $A=\left(a_{n k}\right) \in\left(c s_{0}: \ell_{p}\right)$ if and only if

$$
\sup _{K} \sum_{n}\left|\sum_{k \in K}\left(a_{n k}-a_{n, k+1}\right)\right|^{p}<\infty \quad(1<p<\infty) .
$$

Lemma 29. Consider $A=\left(a_{n k}\right) \in\left(c s: \ell_{p}\right)$ if and only if

$$
\sup _{K} \sum_{n}\left|\sum_{k \in K}\left(a_{n k}-a_{n, k-1}\right)\right|^{p}<\infty \quad(1<p<\infty) .
$$

Lemma 30. Consider $A=\left(a_{n k}\right) \in\left(b s: \ell_{p}\right)$ if and only if (43) and (65) hold.

Now, we give the following results on the matrix transformations.
Theorem 31. (i) Consider $A=\left(a_{n k}\right) \in\left(c s^{\lambda}: \ell_{\infty}\right)$ if and only if

$$
\begin{aligned}
& \sup _{n} \sum_{k=0}^{\infty}\left|\widetilde{a}_{n k}-\widetilde{a}_{n, k-1}\right|<\infty, \\
& \sup _{k}\left|\frac{\lambda_{k}}{\lambda_{k}-\lambda_{k-1}} a_{n k}\right|<\infty .
\end{aligned}
$$

(ii) Consider $A=\left(a_{n \mathrm{k}}\right) \in\left(c s_{0}^{\lambda}: \ell_{\infty}\right)$ if and only if (68) holds and

$$
\sup _{n} \sum_{k=0}^{\infty}\left|\widetilde{a}_{n k}-\widetilde{a}_{n, k+1}\right|<\infty
$$

(iii) Consider $A=\left(a_{n k}\right) \in\left(b s^{\lambda}: \ell_{\infty}\right)$ if and only if (69) holds and

$$
\begin{aligned}
\lim _{k \rightarrow \infty} \frac{\lambda_{k}}{\lambda_{k}-\lambda_{k-1}} a_{n k} & =0, \\
\lim _{k} \tilde{a}_{n k} & =0 .
\end{aligned}
$$

Proof. Suppose that conditions (67) and (68) hold and take any $x=\left(x_{k}\right) \in c s^{\lambda}$. Then, we have by Theorem 23 that $\left(a_{n k}\right)_{k=0}^{\infty} \in\left(c s^{\lambda}\right)^{\beta}$ for all $n \in \mathbf{N}$ and this implies the existence of the $A$-transform of $x$; that is, $A x$ exists. Further, it is clear that the associated sequence $y=\left(y_{k}\right)$ is in $c s$ and hence $y \in c_{0}$.

Let us now consider the following equality derived by using relation (8) from the $m$ th partial sum of the series $\sum_{k} a_{n k} x_{k}$ :

$$
\sum_{k=0}^{m} a_{n k} x_{k}=\sum_{k=0}^{m-1} \widetilde{a}_{n k} y_{k}+\frac{\lambda_{m}}{\lambda_{m}-\lambda_{m-1}} a_{n m} y_{m}
$$

$(\forall n, m \in \mathbf{N})$.

Therefore, by using (67) and (68), from (71) as $m \rightarrow \infty$, we obtain that

$$
\sum_{k} a_{n k} x_{k}=\sum_{k} \widetilde{a}_{n k} y_{k} \quad \forall n \in \mathbf{N} .
$$

Further, since the matrix $\widetilde{A}=\left(\widetilde{a}_{n k}\right)$ is in the class $\left(c s: \ell_{\infty}\right)$ by Lemma 19 and (67), we have $\widetilde{A} y \in \ell_{\infty}$. Therefore, we deduce from (1) and (72) that $A x \in \ell_{\infty}$ and hence $A \in\left(c s^{\lambda}, \ell_{\infty}\right)$.

Conversely, suppose that $A \in\left(c s^{\lambda}, \ell_{\infty}\right)$. Then $\left(a_{n k}\right)_{k=0}^{\infty} \in$ $\left(c s^{\lambda}\right)^{\beta}$ for all $n \in \mathbf{N}$ and this, with Theorem 23, implies both (68) and

$$
\sum_{k=0}^{\infty}\left|\widetilde{a}_{n k}-\widetilde{a}_{k+1}\right|<\infty \quad \forall n \in \mathbf{N}
$$

which together imply that relation (72) holds for all sequences $x \in c s^{\lambda}$ and $y \in c s$. Further, since $A x \in \ell_{\infty}$ by the hypothesis, we obtain by (72) that $\widetilde{A} y \in \ell_{\infty}$ which shows that $\widetilde{A} \in$ (cs: $\left.\ell_{\infty}\right)$, where $\widetilde{A}=\left(\widetilde{a}_{n k}\right)$. Hence, the necessity of (67) is immediate by (48). This concludes the proof of part (i).

Since parts (ii) and (iii) can be proved similarly, we omit their proofs. 
Corollary 32. (i) Consider $A=\left(a_{n k}\right) \in\left(c s^{\lambda}: c\right)$ if and only if (68) and (69) hold and

$$
\lim _{n \rightarrow \infty} \widetilde{a}_{n k} \text { exists. }
$$

(ii) Consider $A=\left(a_{n k}\right) \in\left(c s_{0}^{\lambda}: c\right)$ if and only if (68) and (69) hold and

$$
\lim _{n}\left(\tilde{a}_{n k}-\tilde{a}_{n, k+1}\right) \quad \text { exists. }
$$

(iii) Consider $A=\left(a_{n k}\right) \in\left(b s^{\lambda}: c\right)$ if and only if (70) and (74) hold and

$$
\sum_{k}\left|\tilde{a}_{n k}-\tilde{a}_{n, k-1}\right| \text { convergent. }
$$

Corollary 33. (i) Consider $A=\left(a_{n k}\right) \in\left(c s^{\lambda}: c_{0}\right)$ if and only if (68) and (69) hold and

$$
\lim _{n} \tilde{a}_{n k}=0 .
$$

(ii) Consider $A=\left(a_{n k}\right) \in\left(c s_{0}^{\lambda}: c_{0}\right)$ if and only if (68) and (69) hold and

$$
\lim _{n}\left(\widetilde{a}_{n k}-\tilde{a}_{n, k+1}\right)=0 .
$$
and

(iii) Consider $A=\left(a_{n k}\right) \in\left(b s^{\lambda}: c_{0}\right)$ if and only if (70) hold

$$
\lim _{n} \sum_{k}\left|\widetilde{a}_{n k}-\tilde{a}_{n, k+1}\right|=0
$$

Corollary 34. (i) Consider $A=\left(a_{n k}\right) \in\left(c s^{\lambda}: \ell_{1}\right)$ if and only if (68) holds and

$$
\begin{array}{r}
\sum_{k}\left|\tilde{a}_{n k}-\tilde{a}_{n, k+1}\right|<\infty, \\
\sup _{N, K \in \mathscr{F}}\left|\sum_{n \in N} \sum_{k \in K}\left(\tilde{a}_{n k}-\tilde{a}_{n, k-1}\right)\right|<\infty .
\end{array}
$$

(ii) Consider $A=\left(a_{n k}\right) \in\left(c s_{0}^{\lambda}: \ell_{1}\right)$ if and only if (68) and (80) hold and

$$
\sup _{N, K \in \mathscr{F}}\left|\sum_{n \in N} \sum_{k \in K}\left(\tilde{a}_{n k}-\tilde{a}_{n, k+1}\right)\right|<\infty .
$$

(iii) Consider $A=\left(a_{n k}\right) \in\left(b s^{\lambda}: \ell_{1}\right)$ if and only if (70), (80), and (82) hold.

Corollary 35. (i) Consider $A=\left(a_{n k}\right) \in\left(c s^{\lambda}: \ell_{p}\right)$ if and only if (68) and (80) hold and

$$
\sup _{K \in \mathscr{F}} \sum_{n}\left|\sum_{k \in K}\left(\tilde{a}_{n k}-\tilde{a}_{n, k-1}\right)\right|^{p}<\infty .
$$

(ii) Consider $A=\left(a_{n k}\right) \in\left(c s_{0}^{\lambda}: \ell_{p}\right)$ if and only if (68) and (80) hold and

$$
\sup _{K \in \mathscr{F}} \sum_{n}\left|\sum_{k \in K}\left(\tilde{a}_{n k}-\tilde{a}_{n, k+1}\right)\right|^{p}<\infty .
$$

(iii) Consider $A=\left(a_{n k}\right) \in\left(b s^{\lambda}: \ell_{p}\right)$ if and only if (70), (80), and (84) hold.
Since Corollaries 32, 33, 34, and 35 can be proved similarly with Theorem 31, we omit their proofs.

\section{Some Geometric Properties of the Spaces $c s^{\lambda}, c s_{0}^{\lambda}$, and $b s^{\lambda}$}

In this section, we investigate some geometric properties for the sequence spaces $c s^{\lambda}$ and $b s^{\lambda}$.

Let $(X,\|\cdot\|)$ be a normed linear space, and let $S(X)$ and $B(X)$ be the unit sphere and unit ball of $X$ (for the brevity $X=(X,\|\cdot\|))$, respectively. Consider Clarkson's modulus of convexity (Clarkson [16] and Day [17]) defined by

$$
\begin{aligned}
& \delta_{X}(\epsilon) \\
& \quad=\inf \left\{1-\frac{\|x+y\|}{2} ; x, y \in S(X),\|x-y\|=\epsilon\right\},
\end{aligned}
$$

where $0 \leq \epsilon \leq 2$. The inequality $\delta_{X}>0$ for all $\epsilon \in(0,2]$ characterizes the uniformly convex spaces.

In [18], Gurarî̀s modulus of convexity is defined by

$$
\begin{aligned}
& \beta_{x}(\epsilon)=\inf \{1 \\
& -\inf _{\alpha \in[0,1]}\|\alpha x+(1-\alpha) y\| ; x, y \in S(X),\|x-y\| \\
& =\epsilon\},
\end{aligned}
$$

where $0 \leq \epsilon \leq 2$. It is easily shown that $\delta_{X}(\epsilon) \leq \beta_{x}(\epsilon) \leq$ $2 \delta_{X}(\epsilon)$ for any $0 \leq \epsilon \leq 2$. Also if $0<\beta_{x}(\epsilon)<1$, then $X$ is uniformly convex, and if $\beta_{x}(\epsilon)<1$, then $X$ is strictly convex. Opial property [19] states that

$$
\text { if } x_{n} \stackrel{w}{\longrightarrow} 0 \text {, }
$$$$
\text { then } \limsup _{n \rightarrow \infty}\left\|x_{n}\right\|<\limsup _{n \rightarrow \infty}\left\|x_{n}-x\right\| \quad \forall x \in X, x \neq 0 \text {. }
$$

If the strict inequality becomes $\leq$, this condition becomes a nonstrict Opial property.

The coefficient $R(X)$, introduced by García-Falset [20], is defined as

$$
\begin{aligned}
& R(X):=\sup \left\{\liminf _{n \rightarrow \infty}\left\|x_{n}-x\right\|: x_{n} \stackrel{w}{\longrightarrow} 0,\left\|x_{n}\right\|\right. \\
& \quad \leq 1 \forall n \in \mathbf{N}, \quad\|x\| \leq 1\} .
\end{aligned}
$$

So $1 \leq R(X) \leq 2$ and it is not hard to see that, in the definition of $R(X)$, "liminf" can be replaced by "limsup." Some values of $R(X)$ are $R\left(c_{0}\right)=1$ and $R\left(\ell_{p}\right)=2^{1 / p}, 1<p<\infty$.

A Banach space $X$ has property $(M)$ if whenever $x_{n} \stackrel{w}{\rightarrow} 0$, then $\limsup _{n \rightarrow \infty}\left\|x_{n}-x\right\|$ is a function of $\|x\|$ only. Property $(M)$ which is introduced by Kalton [21] is equivalent to

$$
\begin{aligned}
\text { if } x_{n} \stackrel{w}{\longrightarrow} 0,\|u\| \leq\|v\|, \\
\text { then } \limsup _{n \rightarrow \infty}\left\|x_{n}+u\right\| \leq \limsup _{n \rightarrow \infty}\left\|x_{n}+v\right\| .
\end{aligned}
$$


Sims [22] introduced a property called weak orthogonality (WORTH) for Banach spaces. A Banach space $X$ is said to have property WORTH if

$$
\begin{aligned}
\text { for every } x_{n} \stackrel{w}{\longrightarrow} 0, x \in X, \\
\qquad \limsup _{n \rightarrow \infty}\left\|x_{n}+x\right\|=\limsup _{n \rightarrow \infty}\left\|x_{n}-x\right\| .
\end{aligned}
$$

It remains unknown if property WORTH implies fixed point property. In many situations, the fixed point property can be easily obtained when we assume, in addition, that the spaces are considered to have the property WORTH.

The following result will be used in our results.

Proposition 36 (see [23, Proposition 2.1]). For the following conditions on a Banach space $X$, one has $(i) \Rightarrow$ (ii) $\Rightarrow$ (iii) $\Rightarrow$ (iv).

(i) $X$ has property $(M)$.

(ii) $X$ has property WORTH.

(iii) If $x_{n} \stackrel{w}{\rightarrow} 0$, then for each $x \in X$ we have that $\limsup _{n \rightarrow \infty}\left\|x_{n}-t x\right\|$ is an increasing function of $t$ on $[0, \infty)$.

(iv) X satisfies the nonstrict Opial property.

In $[24,25]$ it has been shown that $R(X)=1$ implies $X$ has property $(M)$.

It has been shown that Banach space $X$ has property $m_{p}$ (resp., $m_{\infty}$ ) [26] if for all $x \in X$, whenever $x_{n} \stackrel{w}{\rightarrow} 0$,

$$
\begin{aligned}
& \limsup _{n \rightarrow \infty}\left\|x+x_{n}\right\|^{p}=\|x\|^{p}+\limsup _{n \rightarrow \infty}\left\|x_{n}\right\|^{p} \\
& \left(\text { resp., } \limsup _{n \rightarrow \infty}\left\|x+x_{n}\right\|\right. \\
& \left.\quad=\max \left\{\|x\|, \limsup _{n \rightarrow \infty}\left\|x_{n}\right\|\right\}\right) .
\end{aligned}
$$

Clearly the above properties imply property $(M)$ and property $m_{1}$ implies Opial property.

Theorem 37 (see [27]). A Banach space $X$ has property $m_{\infty}$ if and only if $R(X)=1$.

Remark 38 (see [28]). A Banach space $X$ with $R(X)<2$ has the weak fixed point property.

Now, let us give our first theorem in this section.

Theorem 39. Gurari's modulus of convexity for the normed spaces $c s^{\lambda}$ and $b s^{\lambda}$ is

$$
\beta_{c s^{\lambda}}(\epsilon)=\beta_{b s^{\lambda}}(\epsilon) \leq 1-|1-\epsilon|,
$$

where $0 \leq \epsilon \leq 2$.

Proof. Assume $x \in c s^{\lambda}$. Then we have

$$
\|x\|_{c s^{\lambda}}=\|x\|_{b s^{\lambda}}=\|\Lambda x\|_{b s}=\sup _{m}\left|\sum_{n=0}^{m}(\Lambda x)_{n}\right| .
$$

Let $0 \leq \epsilon \leq 2$ and consider the following sequences:

$$
\begin{aligned}
& x=\left(x_{n}\right)=\left(\widehat{\Lambda}(1), \widehat{\Lambda}\left(-\frac{\epsilon}{2}\right), \widehat{\Lambda}\left(\frac{\epsilon}{2}\right), 0,0, \ldots\right), \\
& y=\left(y_{n}\right)=\left(\widehat{\Lambda}(1), 0, \widehat{\Lambda}\left(-\frac{\epsilon}{2}\right), \widehat{\Lambda}\left(-\frac{\epsilon}{2}\right), 0, \ldots\right),
\end{aligned}
$$

where $\widehat{\Lambda}$ is the inverse of the matrix $\Lambda$. Since $z_{n}=(\Lambda x)_{n}$ and $t_{n}=(\Lambda y)_{n}$, we have

$$
\begin{aligned}
& z=\left(z_{n}\right)=\left(1,-\frac{\epsilon}{2}, \frac{\epsilon}{2}, 0,0, \ldots\right), \\
& y=\left(y_{n}\right)=\left(1,0,-\frac{\epsilon}{2},-\frac{\epsilon}{2}, 0, \ldots\right) .
\end{aligned}
$$

By using sequences given above, we obtain the following equalities:

$$
\begin{aligned}
\|x\|_{b s^{\lambda}} & =\|\Lambda x\|_{b s}=\sup _{m}\left|\sum_{n=0}^{m}(\Lambda x)_{n}\right|=1, \\
\|y\|_{b s^{\lambda}} & =\|\Lambda y\|_{b s}=\sup _{m}\left|\sum_{n=0}^{m}(\Lambda y)_{n}\right|=1, \\
\|x-y\|_{b s^{\lambda}} & =\|\Lambda x-\Lambda y\|_{b s} \\
& =\sup _{m}\left|\sum_{n=0}^{m}\left((\Lambda x)_{n}-(\Lambda y)_{n}\right)\right|=\epsilon .
\end{aligned}
$$

To complete the conditions of $c s^{\lambda}$ or $b s^{\lambda}$ for Gurarìs modulus of convexity, it remains to evaluate the infimum of $\| \alpha x+(1-$ a) $y \|_{b s^{\lambda}}$ for $0 \leq \alpha \leq 1$. We have

$$
\begin{aligned}
\inf _{0 \leq \alpha \leq 1} & \|\alpha x+(1-\alpha) y\|_{b s^{\lambda}} \\
& =\inf _{0 \leq \alpha \leq 1}\|\alpha \Lambda x+(1-\alpha) \Lambda y\|_{b s} \\
& =\inf _{0 \leq \alpha \leq 1}\left\|\left(1,-\frac{\alpha \epsilon}{2}, \alpha \epsilon-\frac{\epsilon}{2},-\frac{\epsilon}{2}+\frac{\alpha \epsilon}{2}, 0,0, \ldots\right)\right\|_{b s} \\
& =\inf _{0 \leq \alpha \leq 1}|1-\epsilon|=|1-\epsilon| .
\end{aligned}
$$

Consequently we get

$$
\beta_{c s^{\lambda}}(\epsilon)=\beta_{b s^{\lambda}}(\epsilon) \leq 1-|1-\epsilon| .
$$

This is the desired result. Thereby the proof is completed.

Corollary 40. Since $\delta_{c s^{\lambda}}(\epsilon) \leq \beta_{c s^{\lambda}}(\epsilon) \leq 2 \delta_{c s^{\lambda}}(\epsilon)$ and $\delta_{c s^{\lambda}}(\epsilon)=$ $\delta_{b s^{\lambda}}(\epsilon)=0$ for $0 \leq \epsilon \leq 2, \beta_{c s^{\lambda}}(\epsilon)=0<1$ and hence $c^{\lambda}$ and $b s^{\lambda}$ are strictly convex.

Theorem 41. Consider $R(X)=1$ if $X \in\left\{c s^{\lambda}, c s_{0}^{\lambda}\right\}$.

Proof. In $c s^{\lambda}$ ( $\operatorname{or} c s_{0}^{\lambda}$ ), we have $e_{\lambda}^{(n)} \in S(X)$ and $e_{\lambda}^{(n)} \stackrel{w}{\rightarrow} 0$, where $\left(e_{\lambda}^{(n)}\right)$ is the standard basis. Since $e_{\lambda}^{(1)} \in S(X)$, we have

$$
\left\|e_{\lambda}^{(n)}-e_{\lambda}^{(1)}\right\|_{b s^{\lambda}} \stackrel{n \rightarrow \infty}{\longrightarrow} 1 ;
$$

thus $R(X)=1$. 
Now, by Proposition 36 and Theorem 41, we obtain the following results.

Corollary 42. The spaces $c s^{\lambda}$ and $c s_{0}^{\lambda}$ have property $m_{\infty}$ and so the spaces $c s^{\lambda}$ and $c s_{0}^{\lambda}$ have property $(M)$.

Corollary 43. The spaces $c s^{\lambda}$ and $c s_{0}^{\lambda}$ have property WORTH.

Corollary 44. If $x_{n} \stackrel{w}{\rightarrow} 0$, then for each $x \in c s^{\lambda}$ or $c s_{0}^{\lambda}$ we have $\limsup _{n \rightarrow \infty}\left\|x_{n}-t x\right\|$ which is an increasing function of $t$ on $[0, \infty)$.

Corollary 45. The spaces $c s^{\lambda}$ and $c s_{0}^{\lambda}$ satisfy the nonstrict Opial property.

Hence, by Remark 38 and Theorem 41, we have the following result.

Corollary 46. The spaces $c s^{\lambda}$ and $c s_{0}^{\lambda}$ have the weak fixed point property.

\section{Conflict of Interests}

The authors declare that there is no conflict of interests regarding the publication of this paper.

\section{References}

[1] B. Choudhary and S. Nanda, Functional Analysis with Applications, John Wiley \& Sons, New Delhi, India, 1989.

[2] F. Móricz, "On $\Lambda$-strong convergence of numerical sequences and Fourier series," Acta Mathematica Hungarica, vol. 54, no. 3-4, pp. 319-327, 1989.

[3] M. Mursaleen and A. K. Noman, "On the spaces of $\lambda$-convergent and bounded sequences," Thai Journal of Mathematics, vol. 8, no. 2, pp. 311-329, 2010.

[4] M. Mursaleen and A. K. Noman, "Applications of the Hausdorff measure of noncompactness in some sequence spaces of weighted means," Computers \& Mathematics with Applications, vol. 60, no. 5, pp. 1245-1258, 2010.

[5] M. Mursaleen and A. K. Noman, "On some new sequence spaces of non-absolute type related to the spaces $l_{p}$ and $l_{\infty} \mathrm{I}$," Filomat, vol. 25, no. 2, pp. 33-51, 2011.

[6] M. Mursaleen and A. K. Noman, "On some new sequence spaces of non-absolute type related to the spaces $\ell_{p}$ and $\ell_{\infty}$ II," Mathematical Communications, vol. 16, no. 2, pp. 383-398, 2011.

[7] M. Mursaleen and A. K. Noman, "On some new difference sequence spaces of non-absolute type," Mathematical and Computer Modelling, vol. 52, no. 3-4, pp. 603-617, 2010.

[8] V. Karakaya, A. K. Noman, and H. Polat, "On paranormed $\lambda$-sequence spaces of non-absolute type," Mathematical and Computer Modelling, vol. 54, no. 5-6, pp. 1473-1480, 2011.

[9] A. Sönmez and F. Başar, "Generalized difference spaces of nonabsolute type of convergent and null sequences," Abstract and Applied Analysis, vol. 2012, Article ID 435076, 20 pages, 2012.

[10] A. H. Ganie and N. A. Sheikh, "On some new sequence spaces of non-absolute type and matrix transformations," Journal of the Egyptian Mathematical Society, vol. 21, no. 2, pp. 108-114, 2013.
[11] N. A. Sheikh and A. H. Ganie, "On spaces of $\lambda$-convergent sequences and almost convergence," Thai Journal of Mathematics, vol. 11, no. 2, pp. 393-398, 2013.

[12] M. Yeşilkayagil and F. Başar, "On the fine spectrum of the operator defined by the lambda matrix over the spaces of null and convergent sequences," Abstract and Applied Analysis, vol. 2013, Article ID 687393, 13 pages, 2013.

[13] A. Wilansky, Summability Throught Functional Analysis, vol. 85 of North-Holland Mathematics Studies, North-Holland, Oxford, UK, 1984.

[14] E. Malkowsky and V. Rakocevic, "On matrix domains of triangles," Applied Mathematics and Computation, vol. 189, no. 2, pp. 1146-1163, 2007.

[15] M. Stieglitzm and H. Tietz, "Matrixtransformationen von folgenräumen eine ergebnisübersict," Mathematische Zeitschrift, vol. 154, pp. 1-16, 1977.

[16] J. A. Clarkson, "Uniformly convex spaces," Transactions of the American Mathematical Society, vol. 40, no. 3, pp. 396-414, 1936.

[17] M. M. Day, "Uniform convexity in factor and conjugate spaces," Annals of Mathematics, vol. 45, pp. 375-385, 1944.

[18] V. I. Gurarı̆, "On differential properties of the convexity moduli of Banach spaces," Matematicheskie Issledovaniya, vol. 2, pp. 141$148,1967$.

[19] Z. Opial, "Weak convergence of the sequence of successive approximations for nonexpansive mappings," Bulletin of the American Mathematical Society, vol. 73, pp. 591-597, 1967.

[20] J. García-Falset, "Stability and fixed points for nonexpansive mappings," Houston Journal of Mathematics, vol. 20, no. 3, pp. 495-506, 1994.

[21] N. J. Kalton, “M-ideals of compact operators," Illinois Journal of Mathematics, vol. 37, no. 1, pp. 147-169, 1993.

[22] B. Sims, "Orthogonality and fixed points of nonexpansive maps," in Proceedings of the Workshop/Miniconference on Functional Analysis and Optimization, vol. 20, pp. 178-186, Centre for Mathematical Analysis, Australian National University, Canberra, Australia, 1988.

[23] J. García Falset and B. Sims, "Property $(M)$ and the weak fixed point property," Proceedings of the American Mathematical Society, vol. 125, no. 10, pp. 2891-2896, 1997.

[24] T. Dalby, "Relationships between properties that imply the weak fixed point property," Journal of Mathematical Analysis and Applications, vol. 253, no. 2, pp. 578-589, 2001.

[25] B. Sims, "Banach space geometry and the fixed point property," in Recent Advances on Metric Fixed Point Theory (Seville, 1995), vol. 48 of Ciencias, pp. 137-160, Universidad de Sevilla, Seville, Spain, 1995.

[26] H.-K. Xu, G. Marino, and P. Pietramala, "On property $(M)$ and its generalizations," Journal of Mathematical Analysis and Applications, vol. 261, no. 1, pp. 271-281, 2001.

[27] S. Dhompongsa and A. Kaewkhao, "A note on properties that imply the fixed point property," Abstract and Applied Analysis, vol. 2006, Article ID 34959, 12 pages, 2006.

[28] J. G. Falset, "The fixed point property in Banach spaces with the NUS-property," Journal of Mathematical Analysis and Applications, vol. 215, no. 2, pp. 532-542, 1997. 


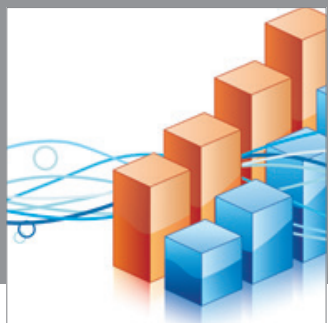

Advances in

Operations Research

mansans

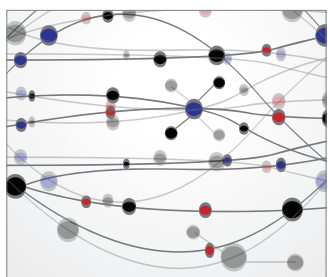

The Scientific World Journal
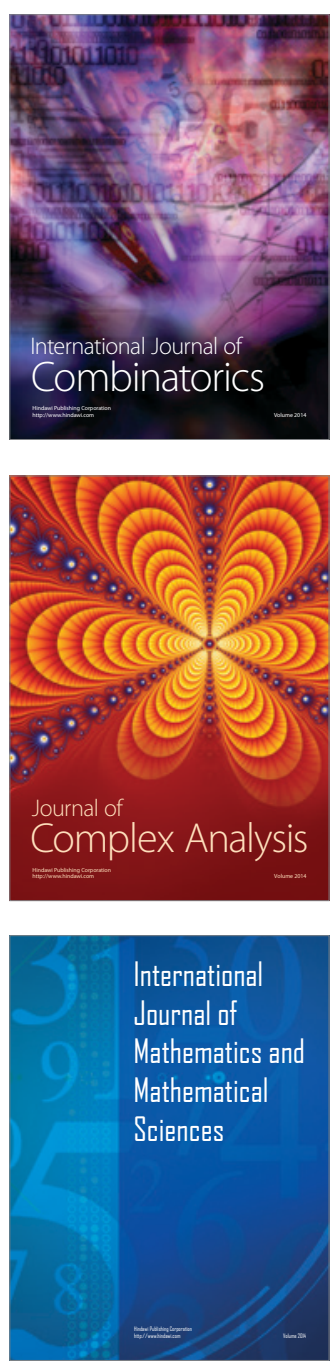
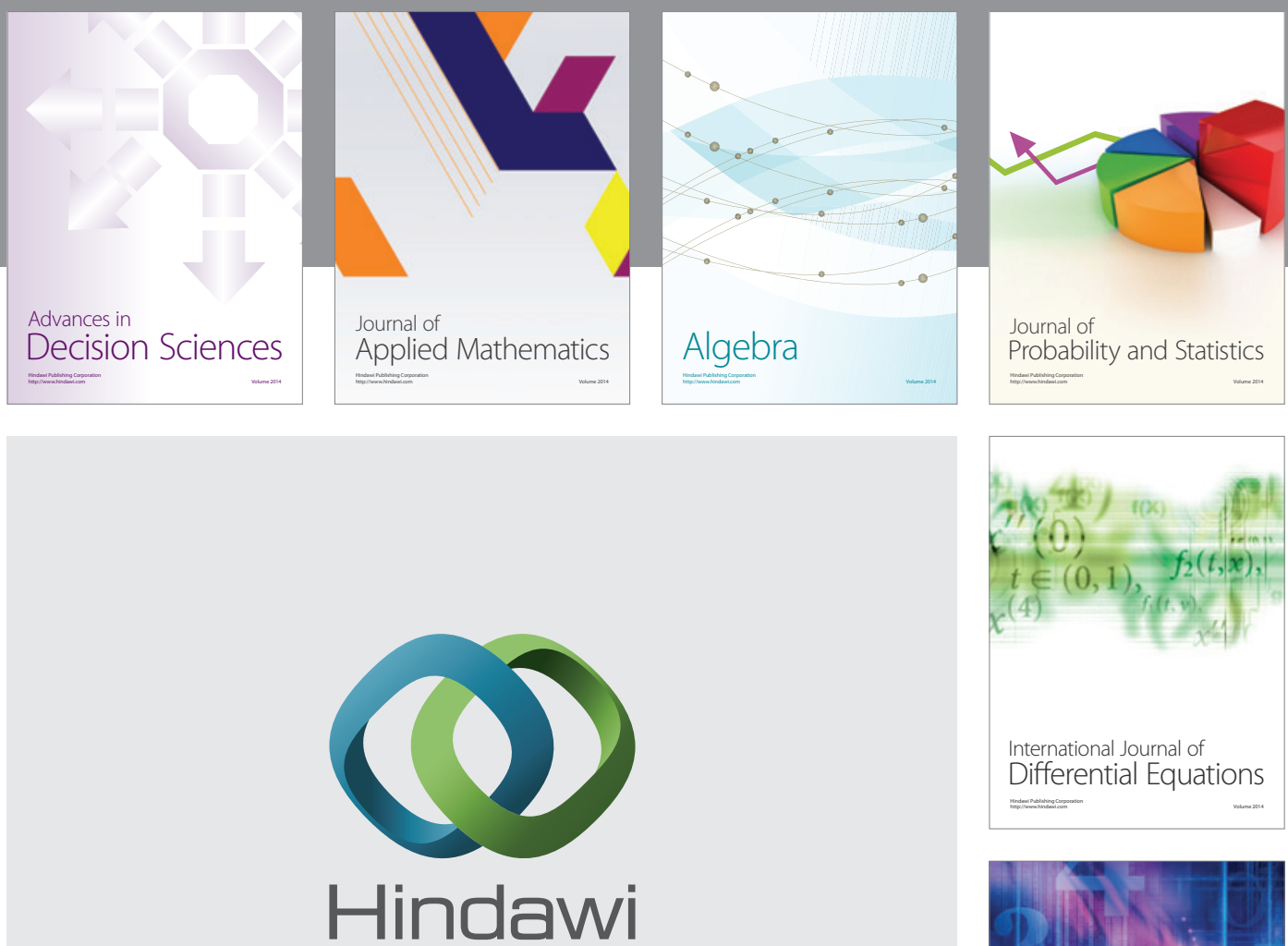

Submit your manuscripts at http://www.hindawi.com
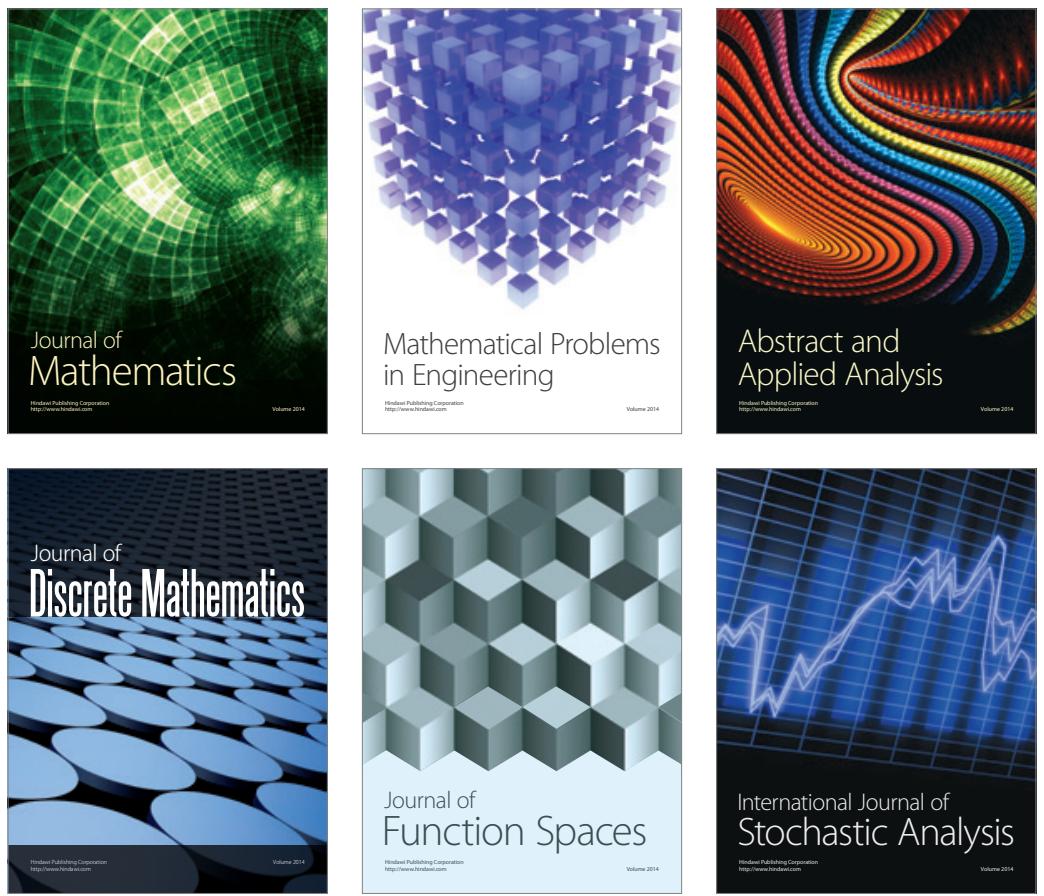

Journal of

Function Spaces

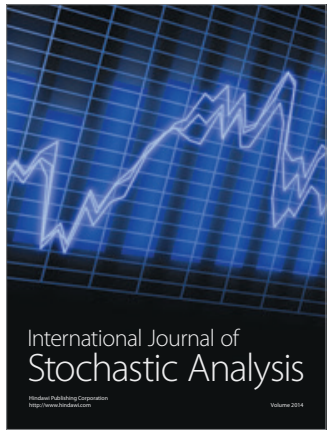

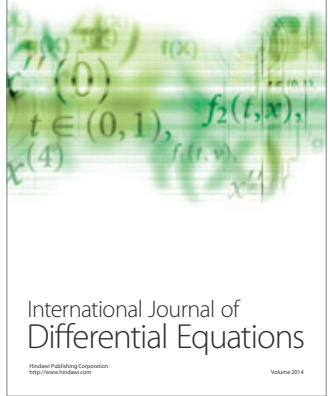
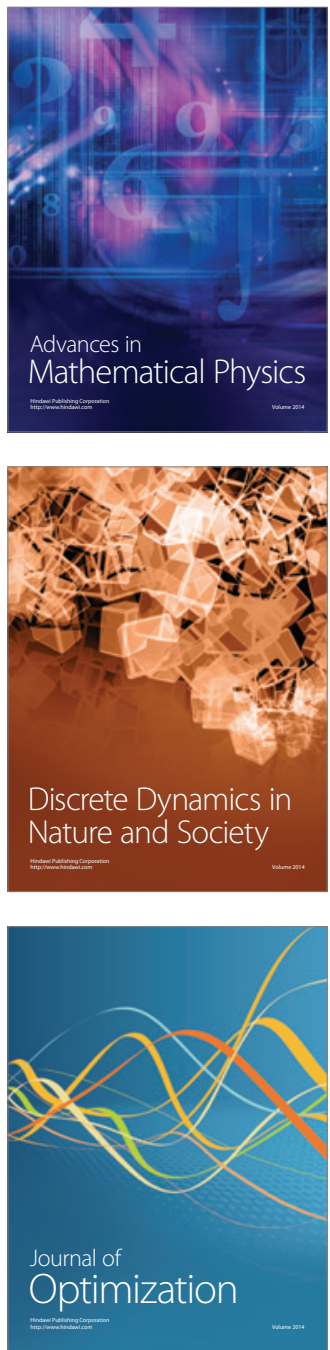